\title{
Panic Disorder: Definitions, Contexts, Neural Correlates and Clinical Strategies
}

\author{
Giulio Perrotta* \\ Department of Criminal and Investigative Psychology Unifeder, Lecturer, International Essayist, Italy
}

*Corresponding author: Giulio Perrotta, Department of Criminal and Investigative Psychology UNIFEDER, Jurist sp. ed SSPL, Lecturer, International Essayist, Italy.

Received Date: July 17, 2019

Published Date: August 14, 2019

\begin{abstract}
Starting from the definition of "panic attacks", we proceeded to list all the forms often mistaken for this disorder, such as anxiety attack, anxiety and terror, using the noso graphic descriptions contained in DSM-V, with particular attention to clinical, neurobiological and therapeutic profiles, concluding the analysis of the possible strategies to be used to finalize the resolutions to the problems deriving from the disorder in question.
\end{abstract}

Keywords: Psychology; Neuroscience; Prefrontal cortex; Frontal lobe; Temporal lobe; Limbic system; Panic attack; Psychotherapy; Psychopharmacology; Benzodiazipines; Antidepressants; Strategic approach

\section{Introduction}

\section{Introductory and contextual aspects about the "anxiety" category}

The definition of anxiety is not unequivocal in the literature (Rachman, 2004), although the descriptive characteristics are well circumscribed and easily identifiable. The American Psychiatric Association, on the definition of anxiety, describes anxiety as: $<<(. .$. the anticipated anticipation of a future danger or negative event, accompanied by feelings of dysphoria or physical symptoms of tension. The elements exposed to risk may belong both to the internal world and to the external world (...) >> (APA, 1994). Similarly, in the Italian Treaty of Psychiatry, precisely in reference to the definition of anxiety, it is referred to as: $<<(.$.$) an emotional$ state with an unpleasant content, associated with a condition of alarm and fear that arises in the absence of danger real and which, however, is disproportionate to any triggering stimuli (...)>> (Perugi-oni, 2002).

However, the idea that it consists of: $<<(. .$.$) a psychophysical$ state characterized by a feeling of apprehension, uncertainty, fear and alarm towards events towards which the subject feels helpless and / or is univocal helpless. It involves a psychic and somatic involvement, associating itself with biological modifications, and involving different systems, among which: neuro-transmittitorial, immune, neuroendocrine (...) >> (Guccione, 2018). $<<(\ldots)$ It represents an essential emotion for the correct functioning of our organism in response to external or internal stimuli, allowing a functional adaptation to environmental demands. The related manifestations of anxiety have a prevalence, in the general population, of $2-4 \%$, a value that rises to $20 \%$ in the non-psychotic psychiatric population. The feeling of anxiety, as well as, in an extremely polymorphous way, is felt by every human being, is characterized by a vague, widespread and unpleasant sense of apprehension, often accompanied by somatic symptoms autonomous species (palpitations, tachycardia, tremors, hyperhidrosis, etc.), but also from psychic symptoms and behavioral manifestations (...) >> (Damiani, 2017). Therefore, anxiety is necessary for the development of one's existence; it becomes the source of a morbid condition if the management of it becomes uncontrollable, to the point of undergoing a negative change in one's lifestyle.

$<<(\ldots)$ Anxiety is innate and is part of human nature. It is the normal response of our body that prepares to face what it perceives as a danger. We have the feeling of being vulnerable, even if sometimes we do not understand for sure why. When our ancestors faced the threat of ferocious animals or hostile peoples, the changes that took place in their bodies prepared them for struggle or flight. Nowadays the dangers are of a completely different nature, but 
faced with a situation that frightens us, or that we perceive as threatening, the same changes occur in us at the time. When anxiety is moderate it can be useful, because it puts us on the alert in the face of a difficult situation allowing us to react quickly. It can become a real problem when it is excessive compared to the situation we are facing or lasts too long, to the point that doing the simplest thing can become a huge effort (...) >> (Lavaggi, 2018).

Another study then focused on the identification of the main constructs that cognitive theory associates with anxiety. In detail: $<<(.$.$) the psychopathological constructs of anxiety are:$

1) Disproportionate fear of harm and tendency to negative predictions or catastrophic thought, definable as the tendency on the part of the subject anxious to foresee a wider range of negative consequences than to non-anxious subjects starting from everyday situations and to conceiving the danger inherent in these negative possibilities as essentially unavoidable, irresistible and irreparable.

2) Fear of error or pathological perfectionism, definable as the tendency to emphasize rather the errors and imperfections present in the tasks performed than the positive results, and to fear and foresee that these imperfections inevitably lead to negative and catastrophic consequences.

3) Intolerance of uncertainty, definable as the tendency to think of not being able to emotionally bear the fact of not knowing perfectly all the possible future scenarios and events, of not being able to bear the doubt that among the possible future events there may be some negative ones, even if this possibility is very low, or to fear that, if there are negative possibilities in a certain scenario, these will be those that inevitably or tendentially occur (of course the negative developments are then feared because of point 1 .

4) Negative self-assessment, definable as the tendency to predict catastrophic scenarios deriving directly from a negative evaluation both of one's own practical skills (negative performance self-assessment) and of one's capacity for emotional self-control and recovery in situations of difficulty and stress (negative self-assessment of weakness, fragility.

5) Need for control, definable as the strenuous pursuit and search by the anxious subject of the illusion of absolute certainty that he can prevent all the negative possibilities that he himself continually feared and foreseen in rumination through continuous monitoring and manipulation some aspects and parameters of external and/or internal reality (e.g weight, food and/or fat in eating disorders, intrusive thoughts or external order in obsessive compulsive disorder, etc.).

In our hypothesis, the tendency to control constitutes the above-mentioned and terminal level of the hierarchical architecture of anxiety. This means that we believe that at the bottom of every anxious state there is always ideally the final belief that things tend to go wrong and that a high degree of knowledge and control of reality is necessary to prevent things from going wrong. The other constructs (fear of damage, fear of error, negative self-assessment and fear of uncertainty) are subordinate and not all always present, at least from the theoretical point of view. Fear of damage and negative self-assessment are the most general ones. It is difficult to say, at present, whether they are organized hierarchically or refer to different areas. Hypothetically it could be assumed that the negative self-assessment is feared because it would lead to damage, and therefore the fear of harm is the terminal belief. Likewise, one might think that it is the negative self-assessment of the central belief that fear of harm is only a predicate. Or one might consider that the two concepts are two different dimensions of a single construct, and therefore they are mutually non-hierarchical in relation (...) >> (Sassaroli-Ruggiero, 2002).

The "pathological" anxiety (Massaro, 2011), therefore, can manifest itself in many ways:

1. Distressing and stressful thoughts and sensations.

2. Physical symptoms, such as cardiovascular symptoms (tachycardia, palpitations, extrasystolia, arrhythmia, pain or discomfort in the chest, hypertension or pressure drops, fainting), respiratory (breathlessness, choking sensation, sensation of a lump in the throat, asthma), gastrointestinal (nausea, gastritis, gastroesophageal reflux, diarrhea, irritable bowel syndrome), neuromuscular (shaking sensation tremor, stiffness, paresthesia, contractures, muscle tension, weakness and fatigue), neurological (vertigo, feeling of "empty head" or light, feeling of heeling, trembling and flushing), dermatological (hives, redness or pallor of the face, hyperhidrosis) and urinary (sudden urge to urinate and pollachiuria).

3. Altered behaviors, such as agitation, increase/decrease appetite and avoidance of certain situations.

In summary, when physiological anxiety becomes an abnormal reaction to a normal alarm situation, it then takes on the pathological appearance of one of the anxiety disorders described in the DSM-V and which we will see in the next section. To be straightforward, physiological anxiety is the sensation of not being able to pass a university exam; the pathological version consists in the choice not to present ourselves at the exam session, despite the fact that there is an intense study of several months behind.

"Healthy" anxiety, however, must also be distinguished from other feelings, often confused in the common jargon in terms of terminology. We are talking about fear, anguish, phobia, panic, fear, terror and stress. Let's start with fear: $<<(.$.$) anxiety is distinguished$ from fear because of the lack of a specific and recognizable stimulus that evokes the answer. This difference is underlined by several authors, including Nisita and Petracca, who describe anxiety as "(...) an emotion that anticipates the danger in the absence of a clearly identified object" (2002). Colombo (2001) defines anxiety in a timely manner as an objectless fear, and Rachman (2004), differentiates anxiety from fear, describing the former as a state of increased vigilance and the latter as a consequent emergency reaction to trigger factors (...) >>. 
Fear can therefore be defined as that primordial feeling, present in every mammal, which allows automatic evaluation of a potential threat or danger so perceived, while anxiety is, instead, a more complex response system involving cognitive and emotional factors, behavioral and physiological. On this basis, it seems correct to state that anxiety and fear are physiological and normal responses in all individuals. Not surprisingly, several studies of cognitive neuroscience (Kandle, 2018) have shown beyond any doubt that anxiety states arise from an abnormal control of fear; in particular, starting from the assumption that anxiety is an adaptive state, anxiety disorders have a genetic component and that the anxious disorders are different in intensity, time course and specific symptomatology, the researchers have concluded, also thanks to the use of images of neurovisualization (fMRI), which in the states of fear and anxiety, are called into question the neural circuits that originate in the amygdala; indeed, the activation of the amygdala was recorded in response to the presentation of a stimulus that induces fear, not consciously perceived.

Anguish is the extreme opposite of peace, the fifth extreme essence of dysfunctional anxiety, where the invasiveness, the restlessness and the sense of catastrophe seen and perceived, from a psychodynamic point of view, from the Ego, such as to undermine the ego's ability to control and manage the pressures of the Superego and the id, consisting of a painful emotional state in which there were processes of discharge, capable of creating symptoms (Freud 1925). From this description we derive the general definition, which embodies it in the sense of frustration and psychophysical malaise, a prelude to various pathologies, precisely because this condition remains for a long time, in a subtle and constant way.

In the clinic, we tend to distinguish the "situational or transitory" form (due to a specific circumstance) from the "existential or chronic" form (due to the lack of processing and maturation of the triggering condition). The phobia is the pathological condition that is generated as a result of specific fear and is determined by a situation that is not really dangerous (or at least less dangerous than the subject feels); this because the phobia, unlike fear, is not proportional to the risk to which one is aware of being exposed or believed to be exposed. Fear degenerates deeply, thus provoking unjustified anxiety.

The fear is simply: $<<(\ldots)$ the state of mind of those who fear can occur a harmful, painful or unpleasant event. It arises when a situation that suggests a pleasant effect, joins the possibility of suffering. One is afraid when the hypothesis that the expected pleasure may not occur is considered, however the hope is still present that pleasure comes and covers the thoughts of different and painful hypotheses. It's the case of a person who waits for the beloved/or an appointment. A minimum delay ignites the fear that the pleasure (loved one) may not arrive, together with the frustration and sorrow (pain) that will ensue. When the person arrives, a smile of contentment covers the previous fear (...)>> (Aruta, 2018).

Terror, by contrast: $<<(.$.$) is even more serious than panic$ In terror, the muscles are paralyzed, the fight / flight reaction is entirely inhibited. It arises in extreme danger or pain situations. It is said: "frozen / petrified" by terror. The body deactivates any sensation coming from the periphery to limit the body's sensitivity in the agony that precedes death. It is a withdrawal inward, as in a state of shock. The breath remains paralyzed in the exhalation phase. Terror can precede fainting, in this case life is maintained by the neuro-vegetative system through unconscious processes. If the terror persists for a long period of time, the depersonalization, dissociation of the ego perceived by the bodily processes (...) >> (Aruta, 2018). It can occur both on a conscious level and during the night hours (e.g Night terror).

And finally, the stress. A term widely used in popular jargon to indicate a state of nervousness and low-level anxiety, often connected to the family or work environment. In the literature, stress is universally regarded as the nonspecific psychophysical response of the organism to every request made on it (Selye, 1974, 1976). Based on the duration of the stressful event it is possible to distinguish two categories of stress: if the stimulus occurs only once and has a limited duration, it is called "acute stress"; if instead the source of stress persists over time, the expression "chronic stress" is used.

Furthermore, according to the nature, the stressor (stressful events) is distinguished in distress, as an event that lowers the immune defenses (correlating it to frustration and anxiety), and eustress, which is an event that fosters greater vitality. The generally perceived symptoms depend on the triggering event but can be summarized in physical-somatic (headache, abdominal pain, muscle pain, sensory disturbances, sexual disorders), emotional (tension, anxiety, unhappiness, restlessness), behavioral (feeding impaired sleep disorders, anger, substance abuse) and cognitive impairment (memory and attention deficit, difficulty in problem solving and agitation).

\section{Definition and clinical contexts of panic disorder}

$<$ (...) Panic is an abnormal and uncontrolled reaction to an initially neutral or mildly stressful situation. If, therefore, pathological anxiety, in most cases, is due to the limits that we impose ourselves for some form of fear, and the anguish is the result of a false Self, of an identity that does not belong to us but that we consider ours and that we do not recognize as false, the panic attack is the clinical manifestation of the result of a long-standing anxiety, to which we have never left space for the elaboration and that, in a moment often of apparent banality or serenity, while the ego's defenses are at a minimum, it hits the victim by paralyzing her. It is not by chance that the main symptoms of a panic attack, according to the DSM-5 (which classifies it as an anxiety disorder) are: palpitations, cardiopalmos, or tachycardia, sweating, fine tremors or great tremors, dyspnoea or suffocation, feeling of asphyxiation (lack of air), chest pain or discomfort, abdominal discomfort, discomfort, instability, lightheaded or fainting, derealization (feeling of unreality) or depersonalization (being detached from oneself), fear of losing control or going crazy, fear of dying, paresthesia (sensations of numbness or tingling), chills or hot flashes. From the panic attack, which single episode, however, 
should be distinguished the real panic disorder, or the simultaneous presence of multiple, unexpected and recurrent panic attacks and at least one of the attacks must have been preceded by the persistent worry of having other attacks or concerns about the implications of the attack or its consequences (e.g losing control, having a heart attack, "going crazy") or significant alteration of the behavior related to the attacks. The presence or absence of agoraphobia then represents a specification. (...) >> [1].

$<<(.$.$) The experience of anguish frightens, a strong sense of$ air hunger and a "crazy" heart that makes death seem imminent, even without a direct connection with dramatic episodes. This is an experience that from the very beginning debases and conditions life, lived with a profound sense of insecurity and shame, with the terror that can be repeated. Although unpleasant (sometimes extreme), panic attacks are not dangerous. (...) The panic attack, therefore, is the most acute and intense form of anxiety and has the characteristics of a crisis that is consumed in about ten minutes. In general, those who have experienced one or more panic attacks tend to develop fear and worry that the panic attack may occur again and concern about the consequences of the panic attack itself (e.g fear that with the occurrence of a series of panic attacks you can go crazy, lose control, risk a heart attack, etc.); consequently there is a tendency to avoid all a series of situations that are considered by the person as "at risk of panic attack" (e.g avoiding places where panic attacks have already occurred, avoiding places where it is difficult to disengage or go out and be able to return to familiar places, implement behavior aimed at protecting yourself from a possible panic attack, for example when you are away from home try to park very close to reach your vehicle as soon as possible in the case in in which the person should be ill, or in any case take "safety measures" if the panic attack occurs).

The most widespread protective behaviors turn out to be carry with you drugs for anxiety; move only in areas where medical facilities are present; leave home only if accompanied by trusted persons; always keep the emergency exits under control. In general, the person tends to avoid all the situations or places that he considers "anxious", in which the person considers that it is difficult to find an "escape" or to receive help in the event of a panic attack. These "avoidances", if extended to different areas and situations of daily life, are very disabling and constricting for the person who lives them, so much so as to compromise the quality of life: often the fear that develops with respect to the panic attack forces many people who do not drive, for example, for fear that a panic attack occurs while driving and therefore lose control in such a situation, the person arrives at this point to compromise their autonomy; or, again, many people who live in very large urban centers who manage to avoid using public transport, such as the underground, so they will have problems moving around and reaching "important" places such as their workplace, school or even worse, social life is compromised (there is a tendency to renounce meeting friends or in general to leave home, often the person feels shame for the consequences that the panic attack may have or fears that other people might notice it) . Among the most widespread "avoidance behavior" are do not use a car, bus, subway, train or plane; not to attend closed places (e.g cinema); do not move away from areas considered safe (e.g home); do not make physical efforts.

Panic attacks can also be classified on the basis of the conditions in which they occur, i.e. dependent on situations and those that occur spontaneously; the latter occur unexpectedly, while those dependent on the situations occur at precise environmental conditions (e.g staying in crowded places, in the elevator, on the underground, in the car, in places where it is difficult to disengage, etc.), or between these, those generated by internal stimuli (e.g physical sensations such as the acceleration of the heartbeat, the sensation of a lump in the throat, assessing that he is blushing in the face, etc.) often interpreted as anticipatory signs of anxiety and/or 'panic attack, or the person may start to think that the cause is within himself and to have thoughts like: "I'm about to faint!", "I'm going to have a heart attack!", "I'll lose control of myself! ",' I'll go crazy! ",' Oh God, I'm going to die!". Panic attacks can also be classified on the basis of the conditions in which they occur, i.e. situations that occur spontaneously; the latter occur unexpectedly, while those are dependent on situations such as staying in crowded places, in the elevator, in the underground, in the car, in places where it is difficult to disengage, etc.), or between these, those generated by internal stimuli (e.g physical sensations such as the acceleration of the heartbeat, the sensation of a lump in the throat, assessment that is blushing in the face, etc.) "I am going to have a heart attack!", "I'll go to have a heart attack!", "I'll lose control of myself!", "I'll go crazy!", "OH God, I'm going to die!”.

In fact, it happens to experience anxiety and fear as these are "legitimate", "normal" emotions, in the sense that in everyday life situations are experienced that justify the emotion of anxiety or fear that one experiences: e.g, a student before examination test anxiety; before a job interview you experience anxiety; waiting for the results of a clinical examination arouses anxiety; etc. in this sense anxiety has an important function, like all the other emotions that one experiences, which is that of signaling that one of our aims is threatened or compromised; for example, if we are crossing a road and we see a vehicle that comes to meet us at high speed without slowing down as it approaches, we estimate that it would be dangerous for our own life, we feel fear and run to save ourselves; therefore anxiety and fear are emotions that generally indicate a danger for one of our important purpose or objectives.

This means that there is a "normal", and therefore healthy, anxiety that is experienced in circumstances in which it is generally legitimate to experience anxiety, since an important purpose is at stake for the person and anxiety is felt because the person considers that this purpose could be compromised (in the previous examples, the student has the purpose of passing the exam; the young person of the interview to pass it and get the job; the patient who waits for the results hopes for the success of the same), and an anxiety " pathological ", which differs from the first in that it is excessive compared to a real danger (e.g thinking of dying or having a heart attack if you experience anxiety, feeling anxious about being on a 
means of transport, in the meaning that in such cases the situations are not "really dangerous" to justify the reaction of intense anxiety).

Pathological anxiety is therefore excessive compared to a real danger, it is characterized by "avoidance" behaviors, that is to avoid certain situations considered risky for the panic attack, this condition generally compromises the quality of life, as the person who he suffers from it tends to limit activities and habits that he faced calmly before he felt ill. All of this generates a sense of frustration and dissatisfaction with one's life. Anxiety has a series of both cognitive symptoms (listlessness, instability, skidding, mental confusion, feeling of unreality, fear of dying, going crazy, losing control) and physical (nausea, abdominal pain, sweating, palpitations, discomfort or pain in the chest, etc.); the physical symptoms manifest themselves consequently to the physiological changes produced by the adrenaline that enters the bloodstream, as anxiety and fear signal a danger and therefore prepare us physically for a "attack-escape" type reaction (e.g of the machine that we comes against). The anxiety considered pathological is the one that triggers an attack-flight-like reaction, but which does not correspond to a real danger. (...) >> [2].

Although therefore they are unpleasant (and sometimes devastating), panic attacks are not dangerous for life, as much for the conduct of a serene and harmonious social and personal life, even if the feeling of imminent death appears real and concrete. In other cases, these anxiety disorders and depression can coexist (co-morbidities), or depression may arise first and the signs and symptoms of anxiety disorders may occur later. Determining whether these attacks are so severe as to be a disorder is a decision that depends on numerous variables and the doctors diverge in making the diagnosis. The diagnosis of a specific anxiety disorder is largely based on its characteristic signs and symptoms.

A family history of anxiety disorders (except post-traumatic stress disorder) is helpful, as many individuals seem to inherit a predisposition to the same anxiety disorders from which their family members suffer, as well as a general vulnerability to other anxiety disorders. $<<(.$.$) Usually, panic disorder is also associated$ with social anxiety disorder or social phobia, as a pathological condition of discomfort and marked fear that an individual experiences in social situations in which there is the possibility of being judged by others, for fear of being embarrassed, to appear ridiculous or incapable and be humiliated in front of others. The typical symptoms are:

a) Marked fear or anxiety related to one or more social situations in which the individual is exposed to the possible judgment of others, such as being observed or performing in front of others.

b) The individual fears that he will act in such a way as to be criticized or manifest anxiety symptoms that will be negatively evaluated.

c) Feared social situations almost invariably cause fear or anxiety. d) Social situations are avoided or endured with intense fear or anxiety.

e) Fear or anxiety are disproportionate to the real threat posed by the social situation and the socio-cultural context.

f) Fear, anxiety or avoidance are persistent and typically last 6 months or more.

(...) Again, we can find this disorder also associated with agoraphobia, a condition in which the affected subject tries to avoid public places or unfamiliar places, has difficulty leaving home and traveling. The severity of anxiety and avoidant behaviors are variable; Agoraphobia is one of the most debilitating anxiety manifestations, as those who suffer from it often become completely dependent on their home walls or are forced to leave home only when they are accompanied. The object of agoraphobia can be to leave home, enter shops, public places, travel alone on buses, trains or planes; panic attacks can relate to the fear of collapsing or being left without help in public, or derive from the lack of an immediate emergency exit (one of the key features of agoraphobic situations). The fear of the social consequences of a panic crisis due to agoraphobia often itself becomes a further cause of emotional difficulty. The fear of leaving the home and relating to the outside world shows a difficulty in dealing with events, people, new and unknown situations, without that "protection", in this case represented by the family environment, where the individual he does not risk immersing himself in the anonymity of the chaotic crowd. Depending on the personal history of each individual, the connection to his habits and daily safety, his level of risk acceptance and relational uncertainty, the meaning assumed by this phobia will be peculiar and therefore it will be up to the psychotherapist to evaluate the type of care to be taken. In the absence of therapy, agoraphobia can become chronic, although usually with a fluctuating clinical course.

(...) Agoraphobic, panic and social anxiety disorders can evolve over time into a true personality disorder called "avoidance of personality disorder"(...). Those suffering from social anxiety disorder could experience panic attacks, very intense anxiety crises that peak in a short time and are accompanied by the fear of going crazy, losing control or dying. In social phobia, panic attacks always occur on the occasion of social situations where others' judgment is feared, thus differentiating themselves from the panic disorder in which the sudden and unexpected attacks are not necessarily linked to interpersonal contexts. In generalized anxiety disorder, the anxious state is constant and, unlike the social anxiety disorder, also present in contexts that are not linked to the judgment of others. In major depressive disorder, the individual may fear the negative judgment of others because they feel devalued and not worthy of approval and appreciation, while in the condition of social anxiety the fear of a bad evaluation by others is linked to the belief that their behavior are inadequate or your appearance and anxious symptoms are a cause for ridicule.

If the reason for the concern is linked solely and exclusively to a shame related to one's physical appearance or to a particular of one's body, one speaks of a disorder of body dysmorphism and 
not of social anxiety disorder. There are no delusional ideas in this disorder, and most individuals with social anxiety have a good judgment about their beliefs or know that they are disproportionate to reality. Social anxiety and communication deficits are common in the autism spectrum disorder. However, those suffering from social anxiety disorder have an initial impairment in these areas in the cognitive phase, unknown people and places, which disappears if they can become familiar. Finally, in the avoidance of personality disorder there are common characteristics with the social anxiety disorder. In avoidance disorders, avoidance is usually more pronounced and extended than social anxiety and has lasted much longer; however, avoidant personality disorder and social anxiety disorder often occur together. (...) >> [2].

After the first panic attack, there are factors that maintain and feed the problem, hindering the solution: sensitivity to anxiety; effect of inconsistency with the emotion experienced; disinformation; disillusioned beliefs and expectations; missed or attempted solutions; protective and/or avoiding behavior [2]. The difference between "panic attack" and "panic attack disorder" is fundamental: in fact, if in the first case we are talking about one or more rare and sporadic episodes, following a specific stress event, in the second case we are talking instead of a real disorder, structured and disabling, which has as its object the panic attack, defined as a sudden and intense episode with fearful and uncontrolled psychophysical manifestations [3].

\section{Etiology and neural correlates in panic disorder}

The etiopathology has not yet been fully clarified; what emerges from recent studies is the implication of both neurophysiological causes and psychological causes; therefore, the condition is necessarily multifactorial [4-7]. From a physiological point of view, all thoughts and feelings can be conceived as resulting from brain electrochemical processes (in a 2004 study it was discovered that three brain areas, anterior cingulate, posterior cingulate and and in raphe), showed serotonin values lower than $1 / 3$ of the minimum physiological standard); however, this says little about the complex interactions between neurotransmitters and neuromodulators in the brain, as well as about anxiety and the normal and pathological state of alarm.

From a psychological point of view, however, panic attacks and panic disorder are considered a response to environmental stressors, such as the interruption of a significant relationship or exposure to a potentially lethal disaster. Even certain physiopathological factors, such as hyperthyroidism, asthma, immune and allergic dysfunctions, use of narcotic and alcoholic substances, but also as lactic acid, sodium bicarbonate, carbon dioxide and caffeine, can aggravate the precarious and vulnerable body chemistry, inducing the anxiogenic state and therefore the onset of panic crisis, up to an actual epileptic attack (in subjects already predisposed).

Many researches have led to the hypothesis that a defective or exaggerated transmission within a circuit that includes the hippocampus, various amygdaloid nuclei, the periaquedottal gray substance, the medial pre-vertebral cortex and the cingulate nucleus, various hypothalamic nuclei, the parabrachial nucleus, the core of the solitary tract, the locus coeruleus and the sensory part of the thalamus, may be responsible for the symptoms of panic attacks. Recently, it seems to me that the frontal and insular cortices are beyond the limbic system. In summary, therefore, the brain structures of the prefrontal cortex, the cingulate, the insular and the amygdala-hippocampus complex are involved.

\section{Clinical strategies for the management of the pathological conditions}

Having ascertained that the disorder in question derives from a dysfunction that has biological and psychological connotations, pharmacological and behavioral therapy are the only tools able to face, manage and overcome the panic attack, both acute and chronic. From a pharmacological point of view, the best choice appears to be oriented towards the prescription of anxiolytics and antidepressants (especially those that have an anxiolytic efficacy), in order to prevent or reduce the anticipatory anxiety, phobic avoidance and the frequency and intensity of panic attacks. Numerous classes of antidepressants, including tricyclics, monoamine oxidase inhibitors, selective serotonin reuptake inhibitors, atypical antidepressants, are fully effective; compared to antidepressants, instead, the choice will fall on a specific drug based on the duration of the treatment and the risk of recurrence, so as to intervene also on the intensity of the attacks. Benzodiazepines, however, have a faster effect than antidepressants (often a few minutes) but are more likely to induce physical dependence and side effects, such as drowsiness, ataxia and memory problems. They are therefore useful for symptomatic treatment and need but are not suitable for prolonged use. An important aspect concerns the question linked to drug dependence and dose ineffectiveness if the administration time is prolonged. All aspects to be evaluated during the interview with the psychiatrist, on a case by case basis [8].

The "Mayo clinic" protocols provide for the use of these product classes [9]:

i. "Selective serotonin reuptake inhibitors" (SSRIs). Generally safe with a low risk of serious side effects, SSRI antidepressants are typically recommended as the first choice of medications to treat panic attacks. SSRIs approved by the Food and Drug Administration (FDA) for the treatment of panic disorder include fluoxetine (Prozac), paroxetine (Paxil, Pexeva) and sertraline (Zoloft).

ii. "Serotonin and norepinephrine reuptake inhibitors" (SNRIs). These medications are another class of antidepressants. The SNRI venlafaxine (Effexor XR) is FDA approved for the treatment of panic disorder.

iii. "Benzodiazepines". These sedatives are central nervous system depressants. Benzodiazepines approved by the FDA for the treatment of panic disorder include alprazolam (Xanax) and clonazepam (Klonopin). Benzodiazepines are generally used only on a short-term basis because they can be habit-forming, 
causing mental or physical dependence. These medications are not a good choice if you've had problems with alcohol or drug use. They can also interact with other drugs, causing dangerous side effects.

Genetic susceptibility, functional alterations of brain structures, the neutrophic factor and the level of inflammation are further possible causes or contributory causes of resistance to drug therapy and therefore of delay in healing [10]. From a psychotherapeutic point of view [11], the cognitive-behavioral approach has proved to be widely shared and effective with respect to the problems related to the disorder in question. Therefore, various techniques such as exposure, guided breathing, imaginary verbal strategies, Acceptance and Commitment Therapy (ACT), Eye Movement Desensitization and Reprocessing (EMDR), autogenic training and yoga are strongly recommended. According to Beck's cognitive-behavioral model (2013) it is not the situation itself that is frightening, but the way we interpret it.

Therefore, events do not cause what we feel, but the way we see them and manage them, through our thoughts. This treatment therefore involves helping the patient in a series of steps: paying attention to what one feels, even at the level of bodily sensations, at a given moment; identify which are the thoughts related to the emotion, one's internal dialogue; to practice questioning dysfunctional thoughts and beliefs; replace dysfunctional thoughts and beliefs with thoughts closer to reality and more useful for achieving one's goals; stop avoiding using behavioral techniques such as enteroceptive and in vivo exposure; prevent relapses.

In recent years, however, other therapeutic hypotheses have come into being, such as group therapies (among these, Andrews suggests the use of his seven-point protocol: psychoeducation, panic monitoring, anxiety management techniques, cognitive restructuring, exposure gradual to situations, gradual exposure to physical sensations, relapse prevention) and self-help interventions; again, two other specific protocols for the resolution of this disorder: a) the first, with a psychodynamic approach; the second, with a short strategic approach.

The first protocol [12], conceived by Massimo Fagioli, is based on the assumption that the ego is formed from the moment a human being comes into the world and with it is formed at birth an image of Self which, not yet being identity, it needs continuous confirmation in the relationship with the reference adults, so that it can develop in a valid way and in parallel with the material wellbeing deriving from being nourished and heated. In the newborn, the valid relationship with the mother promotes physiological development, improving over time its ability to see both physical and mental, to the point of acquiring certainty and awareness of oneself, of others and of the world, thus realizing the development of a "true" self".

If the relationship dynamics do not take place in a physiological way, there is the risk of an initially deficient and then pathological evolution of the self-image, which does not allow the newborn to fully realize its human potential. If the mother is attentive and caring for the needs of the newborn but is unable to grasp the needs of internal living and reactions to the world, we can assume that the child will feel confirmed with respect to his own physical reality, but not in his psychic reality. The child, not yet certain of himself, will tend to make a split between mind and body, which at weaning will evolve in a pathological sense as a split between the conscious mind and the unconscious mind. Conscious reality, no longer having an unconscious internal guide, will forge itself on what comes from the outside: thoughts, rules, stereotypes, developing over time a rational modality detached from its own internal sense.

A process that actively contributes to the development of a "false self". The second pathological trait of panic disorder, the alexithymic typology, derives precisely from the split between mind and body which leads to considering only the needs of the body, gradually eliminating the attention towards non-material internal reality and this is why many Patients treated for panic attacks report an absence of dream activity. The psychotherapeutic relationship, with particular reference to group psychotherapy, allows the patient to increase vitality through the interhuman relationship and with it to realize his own image as a human being. He will therefore be able to make the "false self" disappear and to find again that own reality of affection, curiosity and desire for knowledge that will allow him to complete the partial development of the first year of life and to consolidate a certain identity of self and autonomy, therefore able to continue to evolve throughout life.

The second protocol [13], of short strategic matrix, is based on the studies of over thirty years; the whole procedure is based on four fundamental steps, in order to disrupt the pathogenic perceptive-reactive system of the disorder suffered by the patient. From a "strategic" point of view, the effective intervention on anxiety and panic disorders is based on changing the perception of threatening reality. In fact, if one intervenes at a solely symptomatic level, the risk of relapse is very high or even certain. The strategic approach focuses attention on "how" the problem works and is maintained in the present and on which dysfunctional strategies (the "attempted solutions" codes) are implemented to address it. The person is guided through experiences guided by the therapist to build those individual abilities and abilities that allow to manage the problem to overcome it effectively and definitively [14-16].

$<<(.$.$) "The subterfuges of hope are just as ineffective as the$ arguments of reason "(Cioran, 1993) when the heart beats wildly, the breath becomes labored, the body seems to be crossed by a high voltage electric current and the mind runs fast, looking for a solution to those sensations that one cannot explain. The need for help and protection, as well as the attempt to escape from the situation that you just want to stop, prevent any attempt to be able to control yourself and your reactions. Then, suddenly, everything ends, leaving the same feeling of devastation produced by a tsunami, in this psychological case. Until the next time. We have just taken four steps in crippling fear; the one that terrifies, the one that annihilates. But how can it happen that from a natural fear one can arrive at structuring a real disturbance, which the person cannot get rid of? Fear, as our natural endowment, comes before 
and after everything, pushing us to act anticipating the same mind, with speed and precision. At the same time, precisely because of the described characteristics, when it attacks us, it devastates everything else and the reason is wrecked, the fear exceeds itself and from a great resource becomes limit; becomes panic.

Panic as a psychological disorder is a modern diagnostic category, although the characteristic reaction as a response to conditions of extreme threat, namely the defined "panic-fear", is the most archaic of emotions. The WHO (World Health Organization), in 2000, defined panic disorder as the most important existing disease, affecting $20 \%$ of the population. From a nosographic perspective, in the DSM (Diagnostic and Statistical Manual of Mental Disorders), panic attacks were contradictorily included within the category of anxiety disorders. While, from an operational point of view, it turns out that it is not anxiety that triggers fear, but it is fear that triggers the physiological reaction of anxiety, which sharpens more and more with the rise of perception of individual threat, transforming itself thus from functional mechanism of activation to loss of control.

Following this logic, if the activation of anxiety is an effect of the perception of internal or external stimuli to the organism, the privileged ways of care become the management and transformation of perceptions that trigger the subject's reactions in moments of crisis, while the classification of panic attacks among anxiety disorders leads to a distortion of the observation and evaluation of the disorder, indicating as an adequate solution the pharmacological inhibitory therapy of anxiety itself. It is no coincidence that the first false positive in the diagnosis of panic is represented precisely by the generalized anxiety disorder, where in reality there is no total loss of control typical of panic; the alarm status is constant, with an increase in the physiological parameters, which do not reach tilt however.

From the etiological point of view, despite the really rigorous methodology to understand how a pathology works is represented by the type of therapeutic solution able to solve it, most of the times the perspective remains the traditional one that seeks in the past the causes of the present problem. However, during a panic attack, the person is terrified of his own feelings of fear against the threatening stimulus that he will try to fight, as we will see, in this way increasing them; the effect therefore turns into a cause. Therapeutic change can only occur within the present dynamic of problem persistence, thus acting on the way in which the individual perceives threatening stimuli and, reacting to them, instead of managing them functionally, is overwhelmed. The focus of the study is the interaction of the organism with its reality, to which it responds by modifying it and being modified. Panic is defined by many as the most extreme form of fear that, if below a certain threshold it represents a resource that allows alerting the body to dangerous situations, above this limit becomes pathological There are different situations in which the thrill of fear envelops the person in his coils, but the functioning structure of the vicious circle that creates and maintains fear itself, until it becomes panic, is similar.
By analyzing the most usual reactions to a perception of intense fear, some constant redundancies are observed in different people and situations:

a) The attempt to avoid or shun what scares, which makes one feel less and less able to face that monster that assumes ever more gigantic proportions in the mind of those who are afraid.

b) The search for help and protection, which at the same time makes you feel safe, but then, even if we succeed, it will only be a swab that will take effect until next time. This is because there is a kind of delegation to the other in facing the fear that, being an individual perception, can be exorcised only and only by those who hear it.

c) The failed attempt to keep one's physiological reactions under control, which paradoxically loses control, so we get even more agitated.

The repetition over time of this type of interaction increases the perception of fear leading to an exasperation of the physiological parameters that are activated naturally in the presence of threatening stimuli, up to the explosion of the panic. If, on the contrary, we succeed in interrupting these dysfunctional interactions, fear falls within the limits of functionality [14-16]. This last statement was precisely the hypothesis from which Giorgio Nardone and his collaborators took the first steps for the development of specific intervention protocols: if the avoidance, the request for help and the attempted bankruptcy control are really what turns a fear reaction into a panic, so letting a person suffering from this disorder interrupt such response scripts should lead to the extinction of the disorder itself. In 1987 the first application of a specific therapeutic protocol for panic attacks with agoraphobia was carried out, based on a strategic sequence of therapeutic stratagems that created the planned random events, which led the subjects first to experience the corrective emotional experience, for then being gradually exposed to the feared situations, touching the newly acquired capacities with hand.

The first research-intervention published in 1988 (Nardone, 1988) represented the cornerstone of all the work on panic developed in the following decades to date, demonstrating its extraordinary efficacy and therapeutic efficiency in breaking the rigidity of the phobic perceptive-reactive system obsessive dysfunctional. Currently, the therapeutic treatment developed, and thus tested and proven, represents the "best practice" in the treatment of panic attacks, responding to all the established criteria to be able to evaluate, from an epistemological and empirical point of view, the scientific validity and application of a therapeutic intervention model. Specifically: - the therapeutic changes obtained are maintained over time, with the possibility of repercussions of the disorder reduced to a minimum; to test this, the experimental studies conducted with a control group and randomized samples, the video recordings of the therapeutic processes, and the comparison with other therapeutic techniques, i.e. both qualitative and quantitative assessments (efficacy); the 
therapeutic strategy produces results in reasonably short times, months and not years, otherwise the change could be the effect of fortuitous events (efficiency); therapeutic techniques and their processualism can replicate the results on different subjects that present the same pathology (replicability); - during the application, the effects of each single therapeutic maneuver can be predicted within the entire sequence of the model (predictability); the model and all its techniques are constantly taught and transmitted to other colleagues so that they can achieve similar results (transmissibility) by applying them.

Initially, the unlocking maneuvers acted by blocking the request for help and protection through a restructuring aimed at creating a greater fear that inhibited the present one, resuming the observation that a greater fear puts in the cornering, and those who hear it often pull out a winning courage even in the most adverse conditions. To act on avoidant behavior, a series of suggestive prescriptions were created that could distract the subject during exposure to the feared situations (counter-avoidances), such as the prescription of the pirouette and that of the apple (Nardone, 1993; 2003). Finally, to interrupt the attempt to suppress their reactions, the "logbook" was devised, a sort of apparent monitoring of panic episodes, but in reality, aimed at producing emotional detachment.

This, starting from the observation that, when the subject reacts to the frightening situation driven by some reason or stimulus that distracts him from it, he acts without thinking and, only afterwards, he realizes what he has done successfully. Studies on the neurophysiology of panic (Nardone, 2003, 2016) then highlighted two fundamental processes that take place during a panic attack: on the one hand, the phobic perception involves the limbic system (amygdala, hippocampus, locus coeruleus, hypothalamus ...), that reacts in thousandths of a second by immediately conveying a response to the periphery, activating the "flight or fight" reaction, (which is now "freezing"), thanks to the stimulation of the "freezing", autonomic nervous system, in particular of the sympathetic section. On the other hand, after thousandths of seconds, the sensation reaches the cortex, which is responsible for the conscious evaluation of external stimuli and modulates voluntary behavior; for the amygdala to respond to fear reactions, the medial prefrontal cortex must be deactivated.

The problem emerges when the modern mind, therefore the cortex, confuses the healthy mechanism described with something dangerous, realizing itself out of its control, and what frightens most begins to be no longer fear in itself, but the reaction of loss control of the organism, which leads the reason to try to control, and the more it tries to control the more it loses control, up to the physiological tilt of the panic attack. It was therefore necessary to introduce a technique capable of successfully intervening in panic attacks in the absence of a real threatening source, or in those cases in which the frightening threat does not come from outside, but derives from having fear of the fear that triggers the paradoxical escalation to the point of panic. Paradoxically, fear turns into a selffulfilling prophecy without the need for any external triggering situation. The technique of the "worst fantasy", fruit of the constant work of research-intervention in the field and of concrete examples of success of the paradox in history. Think of the stoic courage of Seneca who, condemned to kill himself by cutting his veins with his own hands and having seen his wife suffer the same fate before him, managed to overcome his fear by spending the period before the execution, imagining all the fantasies most terrible about that atrocious horror movie that would inevitably become the protagonist.

Specifically, the technique consists of asking the person to retire every day in a room where no one can disturb her and, getting comfortable, will dim the lights and create a soft atmosphere. She will set an alarm to sound half an hour later and in this half hour she will begin to fall into all the worst fantasies compared to what could happen to her. And, at this time, she will do whatever she wants to do: if she feels like crying, she cries, if she screams from screaming, if she gets her feet banged on the ground, she does it. When the alarm goes off ... stop ... it's all over; take off the alarm, go to wash your face and go back to your usual day. So, the important thing is that for the entire half-hour, whether or not he feels sick, he stays there, sinking into all the worst fantasies that could happen. He does all that he has to do, but when the alarm goes off ... stop ... it's all over. Detach the alarm clock, wash your face and return to your usual day. So, half an hour of daily passion.

The results of the application of the paradoxical injunction to panic (Frankl,1946) are extraordinary: patients induced to descend into all possible worst fantasies with respect to panic, instead of becoming frightened, relax, creating a counter-paradoxical effect (Nardone, Balbi, 2008) with respect to the paradox of escalation from fear to panic, up to sometimes falling asleep. After a rigorous training, which sees the evolution of the technique from half an hour to five minutes to five times a day when the person has to make scheduled appointments to his fears to become familiar with the experience for which the more he seeks the fear and less this it will be presented, it comes to using the technique before doing something feared (looking at fear in the face so that it becomes courage) and when fear unexpectedly appears (touch the ghost when it appears to make it fade).

In 2000, the evaluation study of 3482 treated cases, of which over $70 \%$ suffered from panic attacks, showed a therapeutic efficacy of $95 \%$ and with a duration of treatments reduced to seven sessions. Since then, hundreds of thousands of cases have been successfully treated, with average success rates in international statistics exceeding $85 \%$. But the most astonishing fact is that the patients get rid of the invalidating disorder within 3-6 months and that these results, as the follow-up measurements after the end of the therapies show, are maintained over time in the absence of relapses and symptom shifts.

This thanks to the application of an isomorphic logic to that of the persistence of the problem, therefore non-ordinary, and to a suggestive-persuasive form of communication. Fear, therefore, if pushed, instead of shunned or repressed, becomes saturated with its own excesses (Nardone,2016), becoming the most evident 
demonstration of the fact that "There is no night that does not see the day" (Nardone, 2003). (...) >> [13, 17-23].

\section{Conclusion}

From the latest studies a still rather fragmented picture emerges with respect to the etiological certainties of panic disorder. If compared to the therapies, the protocols are rather precise and functional, operating between those of psychotherapeutic matrix (the cognitive-behavioral approach and the short strategic one) and the pharmacological ones (anxiolytics and SSRIs/SNRIs antidepressants), the causes that trigger the disorder orientate towards a multifactorial nature: genetic, psychophysiological, food and environmental. In particular, neurobiology plays a fundamental role, demonstrating which brain structures are involved: many researches have led to hypothesis that a defective or exaggerated transmission in a circuit that includes the hippocampus, various amygdaloid nuclei, the periaquedottal gray substance, the medial pre-vertebral cortex and the cingulate nucleus, various hypothalamic nuclei, the parabrachial nucleus, the core of the solitary tract, the locus coeruleus and the sensory part of the thalamus, may be responsible for the symptoms of panic attacks. Recently, it seems to me that the frontal and insular cortices are beyond the limbic system. In summary: the brain structures of the prefrontal cortex, the cingulate, the insular and the amygdala-hippocampus complex are involved. Future research must necessarily be oriented towards prevention and better management of symptoms, as despite great efforts in this direction a good proportion of patients is drugresistant (certainly due to genetic factors) or is still relapsing with pathological symptoms.

\section{Acknowledgement}

None.

\section{Conflict of Interest}

No conflict of Interest.

\section{References}

1. Perrotta G (2019) Anxiety disorders: definitions, contexts, neural correlates and strategic therapy. JJ Neur Neurosci 6(1): 046.

2. Perrotta G (2019) Psicologia clinica, Luxco, USA.

3. Sanavio E, Cornoldi C (2017) Psicologia clinica, $3^{\text {rd }}$ (edn.), Manuali Il Mulino, Italy.
4. Rovetto F (2003) Panico Origini, dinamiche, terapie. Milano: Mc Graw Hill, Italy.

5. Chen MH, Tsai SJ (2016) Treatment-resistant panic disorder: clinical significance, concept and management. Prog Neuropsychopharmacol Biol Psychiatry 70: 219-226.

6. Johnson AL, Mc Leish AC, Shear PK, Privitera M (2018) Panic and epilepsy in adults: a Systematic review. Epilepsy Behav 85: 115-119.

7. Engel KR, Obst K, Bandelow B, Dechent P, Gruber O, et al. (2016) Functional MRI activation in response to panic-specific, non-panic aversive, and neutral pictures in patients with panic disorder and healthy controls. Eur Arch Psychiatry Clin Neurosci 266(6): 557-566.

8. Kotzalidis GD, Brugnoli R, Orlandi V, Donnini M, Parmegiani A, et al. (2001) Neurobiology of Panic Attacks, III Psychiatric Clinic, Journal of Psychopathology, University of Rome "La Sapienza".

9. Andrisano C, Chiesa A, Serretti A (2013) Newer antidepressants and panic disorder: A meta-analysis. Int Clin Psychopharmacol 28: 33-45.

10. http://www.mayoclinic.org/diseases-conditions/panic-attacks/ diagnosis-treatment/drc-20376027

11. Gallagher MW, Payne LA, White KS, Shear KM, Woods SW, et al. (2013) Mechanisms of change in cognitive behavioral therapy for panic disorder: the unique effects of self-efficacy and anxiety sensitivity. Behav Res Ther 51(11):767-777.

12. Dario C, Saba R, Testa L (2018) Panic attacks, in Bios Psychè, Adolescence, Lasino doro.

13. Nardone G (2016) The treatment of panic attacks. Milan: Ponte alle Grazie.

14. Nardone G (2003) There is no night that does not see the day. Therapy in a short time for panic attacks. Ponte alle Grazie, Milan.

15. Nardone G (2000) Beyond the limits of fear. Rizzoli, Milano.

16. Nardone G (1993) Fear, Panic, Phobias: therapy in a short time Ponte alle Grazie.

17. Balbi E, Contribution to the Strategic Short Therapy Center of Nardone, Italy.

18. Castelnuovo G, Molinari E, Nardone G, Salvini A (2013) Empirical research in psychotherapy. In: G Nardone, A Salvini (Edt.), International Dictionary of Psychotherapy. Milan: Garzanti.

19. Cioran EM (1993) Syllogisms of bitterness (trad It 1998) Milan: Adelphi.

20. Frankl VE (1946) Logotherapy and existential analysis (trad It 2005) Brescia: Morcelliana, Nardone G (Edt.), Fear, panic, phobias. Milan: Ponte alle Grazie.

21. Nardone G (2000) Beyond the limits of fear. Milan: Rizzoli.

22. Nardone G (2003) There is no night that does not see the day. Milan: Ponte alle Grazie.

23. Nardone G, Watzlawick P (2005) Brief Strategic Therapy: Philosophy, Techniques and Research. New Jersey: Rowman \& Littlefield, Aronson. 